\title{
WEYL TRANSFORMS ASSOCIATED WITH THE RIEMANN-LIOUVILLE OPERATOR
}

\author{
N. B. HAMADI AND L. T. RACHDI
}

Received 4 January 2006; Revised 21 June 2006; Accepted 8 August 2006

For the Riemann-Liouville transform $\mathscr{R}_{\alpha}, \alpha \in \mathbb{R}_{+}$, associated with singular partial differential operators, we define and study the Weyl transforms $W_{\sigma}$ connected with $\mathscr{R}_{\alpha}$, where $\sigma$ is a symbol in $S^{m}, m \in \mathbb{R}$. We give criteria in terms of $\sigma$ for boundedness and compactness of the transform $W_{\sigma}$.

Copyright (c) 2006 Hindawi Publishing Corporation. All rights reserved.

\section{Introduction}

In his book [14], Wong studies the properties of pseudodifferential operators arising in quantum mechanics, first envisaged by Weyl [13], as bounded linear operators on $L^{2}\left(\mathbb{R}^{n}\right)$ (the space of square integrable functions on $\mathbb{R}^{n}$ with respect to the Lebesgue measure). For this reason, M. W. Wong calls the operators treated in his book Weyl transforms.

Here, we consider the singular partial differential operators

$$
\begin{gathered}
\Delta_{1}=\frac{\partial}{\partial x}, \\
\left.\Delta_{2}=\frac{\partial^{2}}{\partial r^{2}}+\frac{2 \alpha+1}{r} \frac{\partial}{\partial r}-\frac{\partial^{2}}{\partial x^{2}}, \quad(r, x) \in\right] 0,+\infty[\times \mathbb{R}, \alpha \geqslant 0 .
\end{gathered}
$$

We associate to $\Delta_{1}$ and $\Delta_{2}$ the Riemann-Liouville transform $\mathscr{R}_{\alpha}$ defined on $\mathscr{C}_{*}\left(\mathbb{R}^{2}\right)$ (the space of continuous functions on $\mathbb{R}^{2}$, even with respect to the first variable) by

$$
\mathscr{R}_{\alpha}(f)(r, x)= \begin{cases}\frac{\alpha}{\pi} \iint_{-1}^{1} f\left(r s \sqrt{1-t^{2}}, x+r t\right)\left(1-t^{2}\right)^{\alpha-1 / 2}\left(1-s^{2}\right)^{\alpha-1} d t d s & \text { if } \alpha>0, \\ \frac{1}{\pi} \int_{-1}^{1} f\left(r \sqrt{1-t^{2}}, x+r t\right) \frac{d t}{\sqrt{1-t^{2}}} & \text { if } \alpha=0 .\end{cases}
$$

For more general integral transforms, we can see [2].

Hindawi Publishing Corporation

International Journal of Mathematics and Mathematical Sciences

Volume 2006, Article ID 94768, Pages 1-19

DOI 10.1155/IJMMS/2006/94768 
The transform $\mathscr{R}_{\alpha}$ generalizes the mean operator defined by

$$
\mathscr{R}_{0}(f)(r, x)=\frac{1}{2 \pi} \int_{0}^{2 \pi} f(r \sin \theta, x+r \cos \theta) d \theta .
$$

The mean operator $\mathscr{R}_{0}$ and its dual play an important role and have many applications, for example, in image processing of the so-called synthetic aperture radar (SAR) data $[5,6]$, or in the linearized inverse scattering problem in acoustics [3].

In [1], we have defined a convolution product and a Fourier transform $\mathscr{F}_{\alpha}$ associated with $\mathscr{R}_{\alpha}$, and, we have established many harmonic analysis results (inversion formula, Paley-Wiener, and Plancherel theorems, etc.).

Using these results, we define and study, in this paper the Weyl transforms associated with $\mathscr{R}_{\alpha}$, we give criteria in terms of symbols to prove the boundedness and compactness of these transforms. To obtain these results, we have first defined the Fourier-Wigner transform associated with the operator $\mathscr{R}_{\alpha}$, and we have established for it an inversion formula.

More precisely, in Section 2, we recall some properties of harmonic analysis for the operator $\mathscr{R}_{\alpha}$. In Section 3, we define the Fourier-Wigner transform associated with $\mathscr{R}_{\alpha}$, study some of its properties, and prove an inversion formula.

In Section 4, we introduce the Weyl transform $W_{\sigma}$ associated with $\mathscr{R}_{\alpha}$, with $\sigma$ a symbol in class $S^{m}$, for $m \in \mathbb{R}$, and we give its connection with the Fourier-Wigner transform. We prove that for $\sigma$ sufficiently smooth, $W_{\sigma}$ is a compact operator from $L^{2}(d \nu)$, the space of square integrable functions on $[0,+\infty[\times \mathbb{R}$, with respect to the measure

$$
d \nu(r, x)=\frac{1}{2^{\alpha} \Gamma(\alpha+1) \sqrt{2 \pi}} r^{2 \alpha+1} d r \otimes d x
$$

into itself.

In Section 5, we define $W_{\sigma}$ for $\sigma$ in a certain space $L^{p}(d \nu \otimes d \gamma)$, with $p \in[1,2]$, and we establish that $W_{\sigma}$ is again a compact operator.

In Section 6, we define $W_{\sigma}$ for $\sigma$ in another function space, and use this to prove in Section 7 that for $p>2$, there exists a function $\sigma \in L^{p}(d \nu \otimes d \gamma)$, with the property that the Weyl transform $W_{\sigma}$ is not bounded on $L^{2}(d \nu)$.

For more Weyl transforms, we can see $[8,15]$.

\section{Riemann-Liouville transform associated with the operators $\Delta_{1}$ and $\Delta_{2}$}

In this section, we recall some properties of the Riemann-Liouville transform that we use in the next sections. For more details, see [1].

For all $(\mu, \lambda) \in \mathbb{C} \times \mathbb{C}$, the system

$$
\begin{gathered}
\Delta_{1} u(r, x)=-i \lambda u(r, x), \\
\Delta_{2} u(r, x)=-\mu^{2} u(r, x), \\
u(0,0)=1, \quad \frac{\partial u}{\partial r}(0, x)=0, \quad \forall x \in \mathbb{R},
\end{gathered}
$$


admits a unique solution given by

$$
\varphi_{\mu, \lambda}(r, x)=j_{\alpha}\left(r \sqrt{\mu^{2}+\lambda^{2}}\right) \exp (-i \lambda x),
$$

where $j_{\alpha}$ is the modified Bessel function defined by

$$
j_{\alpha}(s)=2^{\alpha} \Gamma(\alpha+1) \frac{J_{\alpha}(s)}{s^{\alpha}}=\Gamma(\alpha+1) \sum_{k=0}^{+\infty} \frac{(-1)^{k}}{k ! \Gamma(\alpha+k+1)}\left(\frac{s}{2}\right)^{2 k},
$$

and $J_{\alpha}$ is the Bessel function of first kind and index $\alpha$ (see $\left.[7,12]\right)$.

Moreover, we have

$$
\sup _{(r, x) \in \mathbb{R}^{2}}\left|\varphi_{\mu, \lambda}(r, x)\right|=1 \quad \text { iff }(\mu, \lambda) \in \Gamma,
$$

where $\Gamma$ is the set defined by

$$
\Gamma=\mathbb{R}^{2} \cup\left\{(i \mu, \lambda) ;(\mu, \lambda) \in \mathbb{R}^{2},|\mu| \leqslant|\lambda|\right\} .
$$

Proposition 2.1. The eigenfunction $\varphi_{\mu, \lambda}$ given by (2.2) has the following Mehler integral representation:

$$
\varphi_{\mu, \lambda}(r, x)= \begin{cases}\frac{\alpha}{\pi} \iint_{-1}^{1} \cos \left(\mu r s \sqrt{1-t^{2}}\right) e^{-i \lambda(x+r t)}\left(1-t^{2}\right)^{\alpha-1 / 2}\left(1-s^{2}\right)^{\alpha-1} d t d s & \text { if } \alpha>0, \\ \frac{1}{\pi} \int_{-1}^{1} \cos \left(r \mu \sqrt{1-t^{2}}\right) e^{-i \lambda(x+r t)} \frac{d t}{\sqrt{1-t^{2}}} & \text { if } \alpha=0 .\end{cases}
$$

This result shows that

$$
\varphi_{\mu, \lambda}(r, x)=\mathscr{R}_{\alpha}(\cos (\mu .) \exp (-i \lambda .))(r, x),
$$

where $\mathscr{R}_{\alpha}$ is the Riemann-Liouville transform associated with the operators $\Delta_{1}$ and $\Delta_{2}$, given in the introduction.

We denote by

(i) $\mathscr{C}_{*, c}\left(\mathbb{R}^{2}\right)$ the subspace of $\mathscr{C}_{*}\left(\mathbb{R}^{2}\right)$ consisting of functions with compact support;

(ii) $d \nu(r, x)$ the measure defined on $[0,+\infty[\times \mathbb{R}$ by

$$
d \nu(r, x)=c_{\alpha} r^{2 \alpha+1} d r \otimes d x,
$$

with $c_{\alpha}=1 / \sqrt{2 \pi} 2^{\alpha} \Gamma(\alpha+1)$;

(iii) $L^{p}(d v)$ the space of measurable functions $f$ on $[0,+\infty[\times \mathbb{R}$, satisfying

$$
\begin{gathered}
\|f\|_{p, v}=\left(\int_{\mathbb{R}} \int_{0}^{+\infty}|f(r, x)|^{p} d \nu(r, x)\right)^{1 / p}<+\infty \quad \text { if } p \in[1,+\infty[, \\
\|f\|_{\infty, \nu}=\operatorname{esssup}_{(r, x) \in[0,+\infty[\times \mathbb{R}}|f(r, x)|<+\infty \quad \text { if } p=+\infty ;
\end{gathered}
$$


(iv) $d \gamma(\mu, \lambda)$ the measure defined on $\Gamma$ by

$$
\begin{aligned}
\iint_{\Gamma} f(\mu, \lambda) d \gamma(\mu, \lambda)=c_{\alpha}\{ & \int_{\mathbb{R}} \int_{0}^{+\infty} f(\mu, \lambda)\left(\mu^{2}+\lambda^{2}\right)^{\alpha} \mu d \mu d \lambda \\
& \left.+\int_{\mathbb{R}} \int_{0}^{|\lambda|} f(i \mu, \lambda)\left(\lambda^{2}-\mu^{2}\right)^{\alpha} \mu d \mu d \lambda\right\} ;
\end{aligned}
$$

(v) $L^{p}(d \gamma), p \in[1,+\infty]$, the space of measurable functions on $\Gamma$ satisfying

$$
\begin{gathered}
\|f\|_{p, \gamma}=\left(\iint_{\Gamma}|f(\mu, \lambda)|^{p} d \gamma(\mu, \lambda)\right)^{1 / p}<+\infty \quad \text { if } p \in[1,+\infty[, \\
\|f\|_{\infty, \gamma}=\underset{(\mu, \lambda) \in \Gamma}{\operatorname{essup}}|f(\mu, \lambda)|<+\infty \quad \text { if } p=+\infty .
\end{gathered}
$$

Defintion 2.2. (i) The translation operator associated with Riemann-Liouville transform is defined on $L^{1}(d v)$, for all $(r, x),(s, y) \in[0,+\infty[\times \mathbb{R}$, by

$$
\mathcal{T}_{(r, x)} f(s, y)=\frac{\Gamma(\alpha+1)}{\sqrt{\pi} \Gamma(\alpha+1 / 2)} \int_{0}^{\pi} f\left(\sqrt{r^{2}+s^{2}+2 r s \cos \theta}, x+y\right) \sin ^{2 \alpha} \theta d \theta
$$

(ii) The convolution product associated with the Riemann-Liouville transform of $f, g \in$ $L^{1}(d \nu)$ is defined by

$$
\forall(r, x) \in\left[0,+\infty\left[\times \mathbb{R}, \quad f * g(r, x)=\int_{\mathbb{R}} \int_{0}^{+\infty} \mathcal{T}_{(r,-x)} \check{f}(s, y) g(s, y) d \nu(s, y),\right.\right.
$$

where $\check{f}(s, y)=f(s,-y)$.

We have the following properties.

(i) We have the following product formula:

$$
\mathscr{T}_{(r, x)} \varphi_{\mu, \lambda}(s, y)=\varphi_{\mu, \lambda}(r, x) \varphi_{\mu, \lambda}(s, y) .
$$

(ii) Let $f$ be in $L^{1}(d v)$. Then, for all $(s, y) \in[0,+\infty[\times \mathbb{R}$, we have

$$
\int_{\mathbb{R}} \int_{0}^{\infty} \mathscr{T}_{(s, y)} f(r, x) d \nu(r, x)=\int_{\mathbb{R}} \int_{0}^{\infty} f(r, x) d \nu(r, x) .
$$

(iii) If $f \in L^{p}(d v), 1 \leqslant p \leqslant+\infty$, then for all $(s, y) \in\left[0,+\infty\left[\times \mathbb{R}\right.\right.$, the function $\mathcal{T}_{(s, y)} f$ belongs to $L^{p}(d \nu)$, and we have

$$
\left\|\mathscr{T}_{(s, y)} f\right\|_{p, \nu} \leqslant\|f\|_{p, \nu} .
$$

(iv) For $f, g \in L^{1}(d \nu), f * g$ belongs to $L^{1}(d \nu)$, and the convolution product is commutative and associative.

(v) For $f \in L^{1}(d \nu), g \in L^{p}(d v), 1<p \leqslant+\infty$, the function $f * g \in L^{p}(d \nu)$ and

$$
\|f * g\|_{p, \nu} \leqslant\|f\|_{1, \nu}\|g\|_{p, \nu} .
$$


(vi) For $f, g \in \mathscr{C}_{*, c}\left(\mathbb{R}^{2}\right)$, such that supp $f \subset\left[-a_{1}, a_{1}\right] \times\left[-a_{2}, a_{2}\right]$ and supp $g \subset\left[-b_{1}\right.$, $\left.b_{1}\right] \times\left[-b_{2}, b_{2}\right]$, the function $f * g$ belongs to $\mathscr{b}_{*, c}\left(\mathbb{R}^{2}\right)$ and

$$
\operatorname{supp}(f * g) \subset\left[-\left(a_{1}+b_{1}\right), a_{1}+b_{1}\right] \times\left[-\left(a_{2}+b_{2}\right), a_{2}+b_{2}\right] .
$$

Defintion 2.3. The Fourier transform associated with the Riemann-Liouville operator is defined on $L^{1}(d \nu)$, by

$$
\forall(\mu, \lambda) \in \Gamma, \quad \mathscr{F}_{\alpha}(f)(\mu, \lambda)=\int_{\mathbb{R}} \int_{0}^{+\infty} f(r, x) \varphi_{\mu, \lambda}(r, x) d \nu(r, x),
$$

where $\Gamma$ is the set defined by the relation (2.5).

We have the following properties.

(i) Let $f$ be in $L^{1}(d v)$. For all $(r, x) \in[0,+\infty[\times \mathbb{R}$, we have

$$
\forall(\mu, \lambda) \in \Gamma, \quad \mathscr{F}_{\alpha}\left(\mathscr{T}_{(r,-x)} f\right)(\mu, \lambda)=\varphi_{\mu, \lambda}(r, x) \mathscr{F}_{\alpha}(f)(\mu, \lambda) .
$$

(ii) For $f, g \in L^{1}(d \nu)$, we have

$$
\forall(\mu, \lambda) \in \Gamma, \quad \mathscr{F}_{\alpha}(f * g)(\mu, \lambda)=\mathscr{F}_{\alpha}(f)(\mu, \lambda) \mathscr{F}_{\alpha}(g)(\mu, \lambda) .
$$

(iii) For $f \in L^{1}(d \nu)$, we have

$$
\forall(\mu, \lambda) \in \Gamma, \quad \mathscr{F}_{\alpha}(f)(\mu, \lambda)=\mathrm{B} \circ \tilde{\mathscr{F}}_{\alpha}(f)(\mu, \lambda),
$$

where, for every $(\mu, \lambda) \in \mathbb{R}^{2}$,

$$
\begin{gathered}
\tilde{\mathscr{F}}_{\alpha}(f)(\mu, \lambda)=\int_{\mathbb{R}} \int_{0}^{+\infty} f(r, x) j_{\alpha}(r \mu) \exp (-i \lambda x) d \nu(r, x), \\
\forall(\mu, \lambda) \in \Gamma, \quad \mathrm{B} f(\mu, \lambda)=f\left(\sqrt{\mu^{2}+\lambda^{2}}, \lambda\right) .
\end{gathered}
$$

(iv) For $f \in L^{1}(d v)$ such that $\mathscr{F}_{\alpha}(f) \in L^{1}(d \gamma)$, we have the inversion formula for $\mathscr{F}_{\alpha}$, for almost every $(r, x) \in[0,+\infty[\times \mathbb{R}$,

$$
f(r, x)=\iint_{\Gamma} \mathscr{F}_{\alpha}(f)(\mu, \lambda) \bar{\varphi}_{\mu, \lambda}(r, x) d \gamma(\mu, \lambda) .
$$

Proposition 2.4. Let $f$ be in $L^{p}(d v)$, with $p \in[1,2]$. Then, $\mathscr{F}_{\alpha}(f)$ belongs to $L^{p^{\prime}}(d \gamma)$, with $1 / p+1 / p^{\prime}=1$, and $\left\|\mathscr{F}_{\alpha}(f)\right\|_{p^{\prime}, \gamma} \leqslant\|f\|_{p, \nu}$.

Proof. The mapping $\tilde{\mathscr{F}}_{\alpha}$ given by the relation (2.23) is an isometric isomorphism from $L^{2}(d v)$ onto itself, then $\left\|\widetilde{\mathscr{F}}_{\alpha}(f)\right\|_{2, \nu}=\|f\|_{2, \nu}$.

On the other hand, we have $\left\|\widetilde{\mathscr{F}}_{\alpha}(f)\right\|_{\infty, v} \leqslant\|f\|_{1, \nu}$.

Thus, from these relations and the Riesz-Thorin theorem $[10,11]$, we deduce that for all $f \in L^{p}(d \nu)$, with $p \in[1,2]$, the function $\widetilde{\mathscr{F}}_{\alpha}(f)$ belongs to $L^{p^{\prime}}(d \nu)$, with $p^{\prime}=p /(p-1)$, and we have

$$
\left\|\widetilde{\mathscr{F}}_{\alpha}(f)\right\|_{p^{\prime}, \nu} \leqslant\|f\|_{p, \nu}
$$


We complete the proof by using the fact that

$$
\left\|\mathscr{F}_{\alpha}(f)\right\|_{p^{\prime}, \gamma}=\left\|\widetilde{\mathscr{F}}_{\alpha}(f)\right\|_{p^{\prime}, v}
$$

which is a consequence of the relation (2.22).

We denote by (see $[1,9])$

(i) $\mathscr{S}_{*}\left(\mathbb{R}^{2}\right)$ the space of infinitely differentiable functions on $\mathbb{R}^{2}$ rapidly decreasing together with all their derivatives, even with respect to the first variable;

(ii) $\mathscr{S}_{*}(\Gamma)$ the space of functions $f: \Gamma \rightarrow \mathbb{C}$ infinitely differentiable, even with respect to the first variable and rapidly decreasing together with all their derivatives, that is, for all $k_{1}, k_{2}, k_{3} \in \mathbb{N}$,

$$
\sup _{(\mu, \lambda) \in \Gamma}\left(1+|\mu|^{2}+|\lambda|^{2}\right)^{k_{1}}\left|\left(\frac{\partial}{\partial \mu}\right)^{k_{2}}\left(\frac{\partial}{\partial \lambda}\right)^{k_{3}} f(\mu, \lambda)\right|<+\infty \text {, }
$$

where

$$
\frac{\partial f}{\partial \mu}(\mu, \lambda)= \begin{cases}\frac{\partial}{\partial r}(f(r, \lambda)) & \text { if } \mu=r \in \mathbb{R}, \\ \frac{1}{i} \frac{\partial}{\partial t}(f(i t, \lambda)) & \text { if } \mu=i t,|t| \leqslant|\lambda| .\end{cases}
$$

Each of these spaces is equipped with its usual topology.

Remark 2.5. From [1], the Fourier transform $\mathscr{F}_{\alpha}$ is an isomorphism from $\mathscr{S}_{*}\left(\mathbb{R}^{2}\right)$ onto $\mathscr{S}_{*}(\Gamma)$. The inverse mapping is given by

$$
\forall(r, x) \in \mathbb{R}^{2}, \quad \mathscr{F}_{\alpha}^{-1}(f)(r, x)=\iint_{\Gamma} f(\mu, \lambda) \bar{\varphi}_{\mu, \lambda}(r, x) d \gamma(\mu, \lambda)
$$

\section{Fourier-Wigner transform associated with Riemann-Liouville operator}

Defintion 3.1. The Fourier-Wigner transform associated with the Riemann-Liouville operator is the mapping $V$ defined on $\mathscr{S}_{*}\left(\mathbb{R}^{2}\right) \times \mathscr{S}_{*}\left(\mathbb{R}^{2}\right)$, for all $((r, x),(\mu, \lambda)) \in \mathbb{R}^{2} \times \Gamma$, by

$$
V(f, g)((r, x),(\mu, \lambda))=\int_{\mathbb{R}} \int_{0}^{\infty} f(s, y) \varphi_{\mu, \lambda}(s, y) \mathcal{T}_{(r, x)} g(s, y) d \nu(s, y) .
$$

Remark 3.2. The transform $V$ can also be written in the forms

(i) $V(f, g)((r, x),(\mu, \lambda))=\mathscr{F}_{\alpha}\left(f \mathscr{T}_{(r, x)} g\right)(\mu, \lambda)$;

(ii) $V(f, g)((r, x),(\mu, \lambda))=\check{g} *\left(\varphi_{\mu, \lambda} f\right)(r,-x)$,

where $\check{g}(s, y)=g(s,-y)$ and $*$ is the convolution product given in Definition 2.2.

We denote by

(i) $\mathscr{S}_{*}\left(\mathbb{R}^{2} \times \mathbb{R}^{2}\right)$ the space of infinitely differentiable functions $f((r, x),(s, y))$ on $\mathbb{R}^{2} \times \mathbb{R}^{2}$, even with respect to the variables $r$ and $s$, and rapidly decreasing together with all their derivatives; 
(ii) $\mathscr{S}_{*}\left(\mathbb{R}^{2} \times \Gamma\right)$ the space of infinitely differentiable functions $f((r, x),(\mu, \lambda))$ on $\mathbb{R}^{2} \times$ $\Gamma$, even with respect to the variables $r$ and $\mu$, and rapidly decreasing together with all their derivatives;

(iii) $L^{p}(d \nu \otimes d \nu), 1 \leqslant p \leqslant+\infty$, the space of measurable functions on $([0,+\infty[\times \mathbb{R}) \times$ $([0,+\infty[\times \mathbb{R})$, verifying for $p \in[1,+\infty[$;

$$
\|f\|_{p, \nu \otimes v}=\left(\iint_{\mathbb{R}} \iint_{0}^{+\infty}|f((r, x),(s, y))|^{p} d \nu(r, x) d \nu(s, y)\right)^{1 / p}<+\infty,
$$

for $p=+\infty$,

$$
\|f\|_{\infty, \nu \otimes \nu}=\operatorname{essup}_{(r, x),(s, y) \in[0,+\infty[\times \mathbb{R}}|f((r, x),(s, y))|<+\infty
$$

(iv) $L^{p}(d \nu \otimes d \gamma), 1 \leqslant p \leqslant+\infty$, the space similarly defined (with $d \nu(r, x) d \gamma(\mu, \lambda)$ in the integrand).

Proposition 3.3. (i) The Fourier-Wigner transform $V$ is a bilinear, continuous mapping from $\mathscr{Y}_{*}\left(\mathbb{R}^{2}\right) \times \mathscr{S}_{*}\left(\mathbb{R}^{2}\right)$ into $\mathscr{S}_{*}\left(\mathbb{R}^{2} \times \Gamma\right)$.

(ii) For $p \in] 1,2]$,

$$
\|V(f, g)\|_{p^{\prime}, \nu \otimes \gamma} \leqslant\|f\|_{p, \nu}\|g\|_{p^{\prime}, \nu}
$$

The transform $V$ can be extended to a continuous bilinear operator, denoted also by $V$, from $L^{p}(d v) \times L^{p^{\prime}}(d v)$ into $L^{p^{\prime}}(d \nu \otimes d \gamma)$, where $p^{\prime}=p /(p-1)$ is the conjugate exponent of $p$.

Proof. (i) Let $f, g \in \mathscr{Y}_{*}\left(\mathbb{R}^{2}\right)$, and let $F$ be the function defined on $\mathbb{R}^{2} \times \mathbb{R}^{2}$ by

$$
F((r, x),(s, y))=f(s, y) \mathscr{T}_{(r, x)} g(s, y)
$$

Then, we have for all $(s, y),(\mu, \lambda) \in \mathbb{R}^{2}$,

$$
\tilde{\mathscr{F}}_{\alpha} \otimes I(F)((\mu, \lambda),(s, y))=j_{\alpha}(s \mu) \exp (i \lambda y) f(s, y) \tilde{\mathscr{F}}_{\alpha}(g)(\mu, \lambda)
$$

where $I$ is the identity operator. Since $\tilde{\mathscr{F}}_{\alpha}$ is an isomorphism from $\mathscr{S}_{*}\left(\mathbb{R}^{2}\right)$ onto itself, we deduce that the function $\tilde{\mathscr{F}}_{\alpha} \otimes I(F)$ belongs to the space $\mathscr{S}_{*}\left(\mathbb{R}^{2} \times \mathbb{R}^{2}\right)$ and consequently, $F \in \mathscr{Y}_{*}\left(\mathbb{R}^{2} \times \mathbb{R}^{2}\right)$. Then, (i) follows from the relation

$$
V(f, g)((r, x),(\mu, \lambda))=I \otimes \mathscr{F}_{\alpha}(F)((r, x),(\mu, \lambda)),
$$

and the fact that $\mathscr{F}_{\alpha}$ is an isomorphism from $\mathscr{S}_{*}\left(\mathbb{R}^{2}\right)$ into $\mathscr{Y}_{*}(\Gamma)$.

(ii) We get the result from Remark 3.2(i), Proposition 2.4, Minkowski's inequality for integrals (see [4, page 186]), and from the relation (2.16).

Theorem 3.4. For all $f, g \in \mathscr{Y}_{*}\left(\mathbb{R}^{2}\right),(\mu, \lambda) \in \Gamma$ and $(r, x) \in \mathbb{R}^{2}$,

$$
\mathscr{F}_{\alpha} \otimes \mathscr{F}_{\alpha}^{-1}(V(f, g))((\mu, \lambda),(r, x))=\bar{\varphi}_{\mu, \lambda}(r, x) f(r, x) \mathscr{F}_{\alpha}(g)(\mu, \lambda) .
$$


Proof. This theorem follows from the relations (2.20) and (3.7).

Using the previous theorem and the relation (2.25), we get the following result.

Corollary 3.5. For $f, g \in \mathscr{Y}_{*}\left(\mathbb{R}^{2}\right)$,

(i) for all $(\mu, \lambda) \in \Gamma$,

$$
\int_{\mathbb{R}} \int_{0}^{\infty} \mathscr{F}_{\alpha} \otimes \mathscr{F}_{\alpha}^{-1}(V(f, g))((\mu, \lambda),(r, x)) d \nu(r, x)=\check{\mathscr{F}}_{\alpha}(f)(\mu, \lambda) \mathscr{F}_{\alpha}(g)(\mu, \lambda) ;
$$

(ii) for all $(r, x) \in[0,+\infty[\times \mathbb{R}$,

$$
\iint_{\Gamma} \mathscr{F}_{\alpha} \otimes \mathscr{F}_{\alpha}^{-1}(V(f, g))((\mu, \lambda),(r, x)) d \gamma(\mu, \lambda)=f(r, x) g(r, x) .
$$

Theorem 3.6. Let $f, g \in L^{1}(d \nu) \cap L^{2}(d v)$, such that $c=\int_{\mathbb{R}} \int_{0}^{\infty} g(r, x) d v(r, x) \neq 0$. Then,

$$
\forall(\mu, \lambda) \in \Gamma, \quad \mathscr{F}_{\alpha}(f)(\mu, \lambda)=\frac{1}{c} \int_{\mathbb{R}} \int_{0}^{\infty} V(f, g)((r, x),(\mu, \lambda)) d \nu(r, x) .
$$

Proof. From the relation (3.1), we have for all $(\mu, \lambda) \in \Gamma$,

$$
\begin{aligned}
\int_{\mathbb{R}} \int_{0}^{\infty} V(f, g)((r, x),(\mu, \lambda)) d \nu(r, x) \\
\quad=\int_{\mathbb{R}} \int_{0}^{\infty}\left(\int_{\mathbb{R}} \int_{0}^{\infty} f(s, y) \varphi_{\mu, \lambda}(s, y) \mathscr{T}_{(r, x)} g(s, y) d \nu(s, y)\right) d \nu(r, x) .
\end{aligned}
$$

Then, the result follows from the relation (2.15), Definition 2.3, the fact that

$$
\forall(r, x) \in\left[0,+\infty\left[\times \mathbb{R}, \forall(\mu, \lambda) \in \Gamma, \quad\left|\varphi_{\mu, \lambda}(r, x)\right| \leqslant 1,\right.\right.
$$

and Fubini's theorem.

Corollary 3.7. With the hypothesis of Theorem 3.6, if $\mathscr{F}_{\alpha}(f) \in L^{1}(d \gamma)$, the following inversion formula for the Fourier-Wigner transform $V$ holds:

$$
f(r, x)=\frac{1}{c} \iint_{\Gamma} \bar{\varphi}_{\mu, \lambda}(r, x)\left[\int_{\mathbb{R}} \int_{0}^{\infty} V(f, g)((s, y),(\mu, \lambda)) d \nu(s, y)\right] d \gamma(\mu, \lambda),
$$

for almost every $(r, x) \in \mathbb{R}^{2}$.

\section{Weyl transform associated with Riemann-Liouville operator}

In this section, we introduce and study the Weyl transform and give its connection with the Fourier-Wigner transform. To do this, we must define the class of pseudodifferential operators [14].

Defintion 4.1. Let $m \in \mathbb{R}$. Define $S^{m}$ to be the set of symbols, consisting of all infinitely differentiable functions $\sigma((r, x),(\mu, \lambda))$ on $\mathbb{R}^{2} \times \Gamma$, even with respect to the variables $r$ and $\mu$, such that for all $k_{1}, k_{2}, k_{3}, k_{4} \in \mathbb{N}$, there exists a positive constant $C=C\left(k_{1}, k_{2}, k_{3}, k_{4}, m\right)$ 
satisfying

$$
\left|\left(\frac{\partial}{\partial r}\right)^{k_{1}}\left(\frac{\partial}{\partial x}\right)^{k_{2}}\left(\frac{\partial}{\partial \mu}\right)^{k_{3}}\left(\frac{\partial}{\partial \lambda}\right)^{k_{4}} \sigma((r, x),(\mu, \lambda))\right| \leqslant C\left(1+\mu^{2}+2 \lambda^{2}\right)^{m-\left(k_{3}+k_{4}\right)} .
$$

Defintion 4.2. For $\sigma \in S^{m}, m \in \mathbb{R}$, define the operator $H_{\sigma}$ on $\mathscr{S}_{*}\left(\mathbb{R}^{2}\right) \times \mathscr{S}_{*}\left(\mathbb{R}^{2}\right)$, for all $(r, x) \in \mathbb{R}^{2}$,

$$
\begin{gathered}
H_{\sigma}(f, g)(r, x)=\iint_{\Gamma}\left\{\int_{\mathbb{R}} \int_{0}^{\infty} \sigma((s, y),(\mu, \lambda)) \varphi_{\mu, \lambda}(r, x)\right. \\
\times V(f, g)((s, y),(\mu, \lambda)) d \nu(s, y)\} d \gamma(\mu, \lambda), \\
\mathbb{U}_{\sigma}(f, g)=H_{\sigma}(f, g)(0,0) .
\end{gathered}
$$

Proposition 4.3. Let $\sigma$ be the symbol given by

$$
\forall(r, x) \in \mathbb{R}^{2}, \forall(\mu, \lambda) \in \Gamma, \quad \sigma((r, x),(\mu, \lambda))=-\left(\mu^{2}+\lambda^{2}\right) .
$$

Then for $f, g \in \mathscr{S}_{*}\left(\mathbb{R}^{2}\right)$,

$$
\forall(r, x) \in \mathbb{R}^{2}, \quad \mathrm{H}_{\sigma}(f, g)(r, x)=c \ell_{\alpha} f(r,-x),
$$

where

$$
c=\int_{\mathbb{R}} \int_{0}^{\infty} g(r, x) d \nu(r, x), \quad \ell_{\alpha}=\frac{\partial^{2}}{\partial r^{2}}+\frac{2 \alpha+1}{r} \frac{\partial}{\partial r} .
$$

Proof. From relations (3.1), (4.2) and Fubini's theorem we get, for all $(r, x) \in \mathbb{R}^{2}$,

$$
\begin{aligned}
\mathrm{H}_{\sigma}(f, g)(r, x)=\iint_{\Gamma}-\left(\mu^{2}+\lambda^{2}\right) \varphi_{\mu, \lambda}(r, x)\left\{\int_{\mathbb{R}} \int_{0}^{\infty}\right. & f(t, z) \varphi_{\mu, \lambda}(t, z) \\
& \left.\times\left[\int_{\mathbb{R}} \int_{0}^{\infty} \mathcal{T}_{(t, z)} g(s, y) d \nu(s, y)\right] d \nu(t, z)\right\} d \gamma(\mu, \lambda) .
\end{aligned}
$$

Now, by relation (2.15), it follows that

$$
\mathrm{H}_{\sigma}(f, g)(r, x)=c \iint_{\Gamma}-\left(\mu^{2}+\lambda^{2}\right) \mathscr{F}_{\alpha}(f)(\mu, \lambda) \varphi_{\mu, \lambda}(r, x) d \gamma(\mu, \lambda) .
$$

The result follows from relation (2.25) and the fact that

$$
\forall(\mu, \lambda) \in \Gamma, \quad-\left(\mu^{2}+\lambda^{2}\right) \mathscr{F}_{\alpha}(f)(\mu, \lambda)=\mathscr{F}_{\alpha}\left(\ell_{\alpha} f\right)(\mu, \lambda) .
$$

Defintion 4.4. Let $\sigma \in S^{m}, m<-(\alpha+3 / 2)$. The Weyl transform associated with the Riemann-Liouville operator is the mapping $W_{\sigma}$ defined on $\mathscr{S}_{*}\left(\mathbb{R}^{2}\right)$, for all $(r, x) \in \mathbb{R}^{2}$, by

$$
W_{\sigma}(f)(r, x)=\iint_{\Gamma}\left[\int_{\mathbb{R}} \int_{0}^{\infty} \varphi_{\mu, \lambda}(r, x) \sigma((s, y),(\mu, \lambda)) \mathscr{T}_{(r, x)} f(s, y) d \nu(s, y)\right] d \gamma(\mu, \lambda) .
$$


Theorem 4.5. Let $\sigma \in \mathscr{S}_{*}\left(\mathbb{R}^{2} \times \Gamma\right)$. The Weyl transform $W_{\sigma}$ is a continuous mapping from $\mathscr{S}_{*}\left(\mathbb{R}^{2}\right)$ into itself.

Proof. Let $f \in \mathscr{S}_{*}\left(\mathbb{R}^{2}\right)$, since $\tilde{\mathscr{F}}_{\alpha}$ is an isomorphism from $\mathscr{S}_{*}\left(\mathbb{R}^{2}\right)$ onto itself, and

$$
\forall(\mu, \lambda) \in \mathbb{R}^{2}, \quad \widetilde{\mathscr{F}}_{\alpha}\left(\mathscr{T}_{(r, x)} f\right)(\mu, \lambda)=j_{\alpha}(r \mu) \exp (i \lambda x) \tilde{\mathscr{F}}_{\alpha}(f)(\mu, \lambda),
$$

we deduce that for all $(r, x) \in\left[0,+\infty\left[\times \mathbb{R}\right.\right.$, the function $(s, y) \mapsto \mathscr{T}_{(r, x)} f(s, y)$ belongs to $\mathscr{S}_{*}\left(\mathbb{R}^{2}\right)$. Then, by the inversion formula for $\widetilde{\mathscr{F}}_{\alpha}$, we get, for all $(s, y) \in \mathbb{R}^{2}$;

$$
\mathscr{T}_{(r, x)} f(s, y)=\int_{\mathbb{R}} \int_{0}^{+\infty} j_{\alpha}(r \mu) \exp (i \lambda x) \widetilde{\mathscr{F}}_{\alpha}(f)(\mu, \lambda) j_{\alpha}(s \mu) \exp (i \lambda y) d \nu(\mu, \lambda) .
$$

By Definition 4.4 and Fubini's theorem, we obtain, for all $(r, x) \in \mathbb{R}^{2}$,

$$
\begin{aligned}
& W_{\sigma}(f)(r, x) \\
& \begin{aligned}
\iint_{\Gamma} \varphi_{\mu, \lambda}(r, x)\left[\int_{\mathbb{R}} \int_{0}^{\infty} \widetilde{\mathscr{F}}_{\alpha}(f)(t, z) j_{\alpha}(r t) \exp (i x z)\right. \\
\left.\quad \times\left\{\int_{\mathbb{R}} \int_{0}^{\infty} \sigma((s, y),(\mu, \lambda)) j_{\alpha}(s t) \exp (i y z) d \nu(s, y)\right\} d \nu(t, z)\right] d \gamma(\mu, \lambda) \\
=\iint_{\Gamma} \varphi_{\mu, \lambda}(r, x)\left[\int_{\mathbb{R}} \int_{0}^{\infty} \widetilde{\mathscr{F}}_{\alpha}(f)(t, z) j_{\alpha}(r t) \exp (i x z)\right. \\
\left.\times \widetilde{\mathscr{F}}_{\alpha}^{-1}(\sigma((\cdot, \cdot),(\mu, \lambda)))(t, z) d \nu(t, z)\right] d \gamma(\mu, \lambda) .
\end{aligned}
\end{aligned}
$$

Now, the function

$$
((t, z),(\mu, \lambda)) \longmapsto \widetilde{\mathscr{F}}_{\alpha}^{-1}(\sigma((\cdot, \cdot),(\mu, \lambda)))(t, z)
$$

belongs to $\mathscr{Y}_{*}\left(\mathbb{R}^{2} \times \Gamma\right)$.

On the other hand, the mapping $f \mapsto G_{f}$, given for all $((t, z),(\mu, \lambda)) \in \mathbb{R}^{2} \times \Gamma$ by

$$
G_{f}((t, z),(\mu, \lambda))=\tilde{\mathscr{F}}_{\alpha}(f)(t, z) \tilde{\mathscr{F}}_{\alpha}^{-1}(\sigma((\cdot, \cdot),(\mu, \lambda)))(t, z),
$$

is continuous from $\mathscr{S}_{*}\left(\mathbb{R}^{2}\right)$ into $\mathscr{S}_{*}\left(\mathbb{R}^{2} \times \Gamma\right)$, and for all $(r, x) \in \mathbb{R}^{2}$, we have

$$
\begin{aligned}
W_{\sigma}(f)(r, x) & =\iint_{\Gamma}\left(\int_{\mathbb{R}} \int_{0}^{\infty} G_{f}((t, z),(\mu, \lambda)) j_{\alpha}(r t) \exp (i x z) \bar{\varphi}_{\mu, \lambda}(r,-x) d \nu(t, z)\right) d \gamma(\mu, \lambda) \\
& =\widetilde{\mathscr{F}}_{\alpha}^{-1} \otimes \mathscr{F}_{\alpha}^{-1}\left(G_{f}\right)((r, x),(r,-x)) .
\end{aligned}
$$

Since $\mathscr{F}_{\alpha}^{-1}$ is an isomorphism from $\mathscr{S}_{*}(\Gamma)$ onto $\mathscr{T}_{*}\left(\mathbb{R}^{2}\right)$, we deduce that $\tilde{\mathscr{F}}_{\alpha}^{-1} \otimes \mathscr{F}_{\alpha}^{-1}$ is an isomorphism from $\mathscr{S}_{*}\left(\mathbb{R}^{2} \times \Gamma\right)$ onto $\mathscr{S}_{*}\left(\mathbb{R}^{2} \times \mathbb{R}^{2}\right)$. 
Lemma 4.6. Let $\sigma \in \mathscr{S}_{*}\left(\mathbb{R}^{2} \times \Gamma\right)$. Then, the function $k$ defined on $\mathbb{R}^{2} \times \mathbb{R}^{2}$ by

$$
k((r, x),(s, y))=\iint_{\Gamma} \varphi_{\mu, \lambda}(r, x) \mathscr{T}_{(r,-x)}(\sigma((\cdot, \cdot),(\mu, \lambda)))(s, y) d \gamma(\mu, \lambda)
$$

belongs to $\mathscr{Y}_{*}\left(\mathbb{R}^{2} \times \mathbb{R}^{2}\right)$.

Proof. The function $k$ can be written in the form

$$
k((r, x),(s, y))=\mathscr{T}_{(r,-x)}\left(I \otimes \mathscr{F}_{\alpha}^{-1}(\sigma)((\cdot, \cdot),(r,-x))\right)(s, y) .
$$

Since the Fourier transform $\mathscr{F}_{\alpha}$ is an isomorphism from $\mathscr{S}_{*}\left(\mathbb{R}^{2}\right)$ onto $\mathscr{S}_{*}(\Gamma)$, we deduce that the function $I \otimes \mathscr{F}_{\alpha}^{-1}(\sigma)$ belongs to $\mathscr{Y}_{*}\left(\mathbb{R}^{2} \times \mathbb{R}^{2}\right)$.

Then, the lemma follows from the fact that for all $g \in \mathscr{S}_{*}\left(\mathbb{R}^{2} \times \mathbb{R}^{2}\right)$, the function

$$
((r, x),(s, y)) \longmapsto \mathscr{T}_{(r,-x)}(g((\cdot, \cdot),(r,-x)))(s, y)
$$

belongs to $\mathscr{S}_{*}\left(\mathbb{R}^{2} \times \mathbb{R}^{2}\right)$.

Theorem 4.7. Let $\sigma \in \mathscr{S}_{*}\left(\mathbb{R}^{2} \times \Gamma\right)$.

(i) For all $f \in \mathscr{S}_{*}\left(\mathbb{R}^{2}\right)$,

$$
\forall(r, x) \in \mathbb{R}^{2}, \quad W_{\sigma}(f)(r, x)=\int_{\mathbb{R}} \int_{0}^{\infty} k((r, x),(s, y)) f(s, y) d \nu(s, y) .
$$

(ii) For $f \in \mathscr{Y}_{*}\left(\mathbb{R}^{2}\right)$ and $p, p^{\prime} \in[1,+\infty]$ such that $1 / p+1 / p^{\prime}=1$,

$$
\left\|W_{\sigma}(f)\right\|_{p^{\prime}, v} \leqslant\|k\|_{p^{\prime}, v \otimes v}\|f\|_{p, \nu} .
$$

(iii) For $p \in\left[1,+\infty\left[\right.\right.$, the operator $W_{\sigma}$ can be extended to a bounded operator from $L^{p}(d \nu)$ into $L^{p^{\prime}}(d \nu)$.

In particular

$$
W_{\sigma}: L^{2}(d \nu) \longmapsto L^{2}(d \nu)
$$

is a Hilbert-Schmidt operator, and consequently it is compact.

Proof. (i) Let $f$ be in $\mathscr{S}_{*}\left(\mathbb{R}^{2}\right)$. From Definition 4.4 , for all $(\mu, \lambda) \in \mathbb{R}^{2}$, we have

$$
\begin{aligned}
W_{\sigma}(f)(r, x) & =\iint_{\Gamma}\left(\int_{\mathbb{R}} \int_{0}^{\infty} \varphi_{\mu, \lambda}(r, x) \sigma((s, y),(\mu, \lambda)) \mathcal{T}_{(r, x)} f(s, y) d \nu(s, y)\right) d \gamma(\mu, \lambda) \\
& =\iint_{\Gamma} \varphi_{\mu, \lambda}(r, x)\left(\int_{\mathbb{R}} \int_{0}^{\infty} \sigma((s, y),(\mu, \lambda)) \mathcal{T}_{(r, x)} f(s, y) d \nu(s, y)\right) d \gamma(\mu, \lambda) .
\end{aligned}
$$

Using Fubini's theorem, and the equality

$$
\begin{aligned}
\int_{\mathbb{R}} \int_{0}^{\infty} \sigma((s, y),(\mu, \lambda)) \mathcal{T}_{(r, x)} f(s, y) d \nu(s, y) \\
\quad=\int_{\mathbb{R}} \int_{0}^{\infty} f(s, y) \mathcal{T}_{(r,-x)}(\sigma((\cdot, \cdot),(\mu, \lambda)))(s, y) d \nu(s, y),
\end{aligned}
$$


we get

$$
\begin{aligned}
W_{\sigma}(f)(r, x) & =\int_{\mathbb{R}} \int_{0}^{\infty} f(s, y)\left\{\iint_{\Gamma} \varphi_{\mu, \lambda}(r, x) \mathscr{T}_{(r,-x)}(\sigma((\cdot, \cdot),(\mu, \lambda)))(s, y) d \gamma(\mu, \lambda)\right\} d \nu(s, y) \\
& =\int_{\mathbb{R}} \int_{0}^{\infty} f(s, y) k((r, x),(s, y)) d \nu(s, y) .
\end{aligned}
$$

(ii) follows from (i), Hölder's inequality, and Lemma 4.6.

(iii) From (ii) and the fact that the space $\mathscr{S}_{*}\left(\mathbb{R}^{2}\right)$ is dense in $L^{p}(d \nu), p \in[1,+\infty[$, we deduce that $W_{\sigma}$ can be extended to a continuous mapping from $L^{p}(d \nu)$ into $L^{p^{\prime}}(d \nu)$.

By Lemma 4.6 , the kernel $k$ belongs to $L^{2}(d \nu \otimes d \nu)$, hence $W_{\sigma}$ is a Hilbert-Schmidt operator. In particular, it is compact.

Theorem 4.8. Let $\sigma \in S^{m}, m<-(\alpha+3 / 2)$. For all $f, g \in \mathscr{Y}_{*}\left(\mathbb{R}^{2}\right)$, we have

$$
\mathbb{W}_{\sigma}(f, g)=\left\langle\frac{W_{\sigma}(g)}{\bar{f}}\right\rangle,
$$

where $\langle\cdot / \cdot\rangle$ is the inner product of $L^{2}(d \nu)$.

Proof. From Definition (3.1) and relations (4.2), (4.3), we get

$$
\begin{aligned}
\mathbb{H}_{\sigma}(f, g)=\iint_{\Gamma}\left\{\int _ { \mathbb { R } } \int _ { 0 } ^ { \infty } \sigma ( ( r , x ) , ( \mu , \lambda ) ) \left(\int_{\mathbb{R}} \int_{0}^{\infty}\right.\right. & f(s, y) \varphi_{\mu, \lambda}(s, y) \\
& \left.\left.\times \mathcal{T}_{(r, x)} g(s, y) d \nu(s, y)\right) d \nu(r, x)\right\} d \gamma(\mu, \lambda) .
\end{aligned}
$$

Using Fubini's theorem, we obtain

$$
\begin{array}{r}
\mathbb{H}_{\sigma}(f, g)=\int_{\mathbb{R}} \int_{0}^{\infty} f(s, y)\left\{\int \int _ { \Gamma } \varphi ( \mu , \lambda ) ( s , y ) \left(\int_{\mathbb{R}} \int_{0}^{\infty} \sigma((r, x),(\mu, \lambda))\right.\right. \\
\left.\left.\times \mathscr{T}_{(r, x)} g(s, y) d \nu(r, x)\right) d \gamma(\mu, \lambda)\right\} d \nu(s, y) .
\end{array}
$$

The theorem follows from Definition 4.4 and the fact that for all $((r, x),(s, y)) \in[0$, $+\infty[\times \mathbb{R}$,

$$
\mathscr{T}_{(r, x)} g(s, y)=\mathscr{T}_{(s, y)} g(r, x)
$$

5. Weyl transform associated with symbol in $L^{p}(d \nu \otimes d \gamma), 1 \leqslant p \leqslant 2$

In this section, we will see that relation (4.26) allows us to prove that the Weyl transform with symbol in $L^{p}(d v \otimes d \gamma), 1 \leqslant p \leqslant 2$, is a compact operator. 
We denote by $\mathscr{B}\left(L^{2}(d v)\right)$ the $\mathbb{C}^{*}$-algebra of bounded operators $\psi$ from $L^{2}(d \nu)$ into itself, equipped with the norm

$$
\|\psi\|_{*}=\sup _{\|f\|_{2, \nu}=1}\|\psi(f)\|_{2, v^{*}}
$$

Theorem 5.1. For $p \in[1,2]$, there exists a unique bounded operator $Q$ from $L^{p}(d \nu \otimes d \gamma)$ into $\mathscr{B}\left(L^{2}(d v)\right): \sigma \mapsto Q_{\sigma}$, such that for all $f, g \in \mathscr{Y}_{*}\left(\mathbb{R}^{2}\right)$,

$$
\begin{gathered}
\left\langle\frac{Q_{\sigma}(g)}{\bar{f}}\right\rangle=\iint_{\Gamma}\left(\int_{\mathbb{R}} \int_{0}^{\infty} \sigma((r, x),(\mu, \lambda)) V(f, g)((r, x),(\mu, \lambda)) d \nu(r, x)\right) d \gamma(\mu, \lambda), \\
\left\|Q_{\sigma}\right\|_{*} \leqslant\|\sigma\|_{p, \nu \otimes \gamma .}
\end{gathered}
$$

Proof. (i) The case $p=2$.

Let $\sigma \in \mathscr{S}_{*}\left(\mathbb{R}^{2} \times \Gamma\right)$. For $g \in \mathscr{S}_{*}\left(\mathbb{R}^{2}\right)$, we put $Q_{\sigma}(g)=W_{\sigma}(g)$.

From Theorem 4.8, we obtain

$$
\begin{aligned}
\left\langle\frac{Q_{\sigma}(g)}{\bar{f}}\right\rangle & =\left\langle\frac{W_{\sigma}(g)}{\bar{f}}\right\rangle=\mathbb{H}_{\sigma}(f, g) \\
& =\iint_{\Gamma}\left(\int_{\mathbb{R}} \int_{0}^{\infty} \sigma((r, x),(\mu, \lambda)) V(f, g)((r, x),(\mu, \lambda)) d \nu(r, x)\right) d \gamma(\mu, \lambda) .
\end{aligned}
$$

On the other hand, from Proposition 3.3(ii) and Cauchy-Shwartz inequality, we have

$$
\left|\left\langle\frac{Q_{\sigma}(g)}{\bar{f}}\right\rangle\right| \leqslant\|\sigma\|_{2, \nu \otimes \gamma}\|f\|_{2, \nu}\|g\|_{2, \nu} .
$$

This implies that $Q_{\sigma} \in \mathscr{B}\left(L^{2}(d v)\right)$ and

$$
\left\|Q_{\sigma}\right\|_{*} \leqslant\|\sigma\|_{2, \nu \otimes \gamma}
$$

We complete the proof by using the fact that the space $\mathscr{S}_{*}\left(\mathbb{R}^{2} \times \Gamma\right)$ is dense in $L^{2}(d \nu \otimes d \gamma)$.

(ii) The case $p=1$ can be obtained by the same way.

(iii) Using the cases $p=1, p=2$, and the Riesz-Thorin theorem $[10,11]$, we complete the proof for all $p \in[1,2]$.

Remark 5.2. In the following, the operator $Q_{\sigma}$ will be denoted by $W_{\sigma}$.

Theorem 5.3. For $\sigma \in L^{p}(d \nu \otimes d \gamma), 1 \leqslant p \leqslant 2$, the operator $W_{\sigma}$ from $L^{2}(d \nu)$ into itself is a compact operator.

Proof. Let $\sigma \in L^{p}(d \nu \otimes d \gamma), 1 \leqslant p \leqslant 2$, and let $\left(\sigma_{k}\right)_{k \in \mathbb{N}}$ be a sequence in $\mathscr{Y}_{*}\left(\mathbb{R}^{2} \times \Gamma\right)$, such that

$$
\left\|\sigma_{k}-\sigma\right\|_{p, \nu \otimes \gamma} \underset{k \rightarrow+\infty}{\longrightarrow} 0 .
$$

From relation (5.5), we have $\left\|W_{\sigma_{k}}-W_{\sigma}\right\|_{*} \leqslant\left\|\sigma_{k}-\sigma\right\|_{p, \nu \otimes \gamma}$. This implies that

$$
W_{\sigma_{k}} \underset{k \rightarrow+\infty}{\longrightarrow} W_{\sigma}, \quad \text { in } \mathscr{B}\left(L^{2}(d \nu)\right) \text {. }
$$


But from Theorem 4.7, we know that for all $k \in \mathbb{N}$, the operator $W_{\sigma_{k}}$ is compact, then the result of the theorem follows from the fact that the subspace $\mathscr{K}\left(L^{2}(d v)\right)$ of $\mathscr{B}\left(L^{2}(d v)\right)$ consisting of compact operators is a closed ideal of $\mathscr{B}\left(L^{2}(d \nu)\right)$.

\section{Weyl transform with symbol in $S_{*}^{\prime}\left(\mathbb{R}^{2} \times \Gamma\right)$}

We denote by

(i) $\mathscr{S}_{*}^{\prime}\left(\mathbb{R}^{2}\right)$ the space of tempered distributions on $\mathbb{R}^{2}$, even with respect to the first variable. It is the topological dual of $\mathscr{Y}_{*}\left(\mathbb{R}^{2}\right)$;

(ii) $\mathscr{S}_{*}^{\prime}\left(\mathbb{R}^{2} \times \Gamma\right)$ the space of tempered distributions on $\mathbb{R}^{2} \times \Gamma$, even with respect to the first variables of $\mathbb{R}^{2}$ and $\Gamma$. It is the topological dual of $\mathscr{Y}_{*}\left(\mathbb{R}^{2} \times \Gamma\right)$.

Defintion 6.1. For $\sigma \in \mathscr{S}_{*}^{\prime}\left(\mathbb{R}^{2} \times \Gamma\right)$ and $g \in \mathscr{Y}_{*}\left(\mathbb{R}^{2}\right)$, define the operator $W_{\sigma}(g)$ on $\mathscr{Y}_{*}\left(\mathbb{R}^{2}\right)$, by

$$
\left[W_{\sigma}(g)\right](f)=\sigma(V(f, g)), \quad f \in \mathscr{S}_{*}\left(\mathbb{R}^{2}\right),
$$

where $V$ is the mapping given by (3.1).

Remark 6.2. From Proposition 3.3, it is clear that $W_{\sigma}(g)$ given by $(6.1)$ belongs to $S_{*}^{\prime}\left(\mathbb{R}^{2}\right)$.

For a slowly increasing function $h$ on $\mathbb{R}^{2} \times \Gamma$, we denote by $\sigma_{h}$ the element of $S_{*}^{\prime}\left(\mathbb{R}^{2} \times \Gamma\right)$ defined by

$$
\sigma_{h}(F)=\iint_{\Gamma} \int_{\mathbb{R}} \int_{0}^{\infty} F((r, x),(\mu, \lambda)) h((r, x),(\mu, \lambda)) d \nu(r, x) d \gamma(\mu, \lambda) .
$$

Then, we have the following.

Proposition 6.3. Let $\sigma_{1} \in S_{*}^{\prime}\left(\mathbb{R}^{2} \times \Gamma\right)$, given by the function equal to 1 . One has

$$
W_{\sigma_{1}}(g)=c \delta
$$

where $c=\int_{\mathbb{R}} \int_{0}^{\infty} g(r, x) d \nu(r, x)$ and $\delta$ is the Dirac distribution at $(0,0)$.

Proof. By relation (6.1), we have for all $f$ in $\mathscr{Y}_{*}\left(\mathbb{R}^{2}\right)$,

$$
\begin{aligned}
{\left[W_{\sigma_{1}}(g)\right](f) } & =\sigma_{1}(V(f, g)), \\
& =\iint_{\Gamma}\left(\int_{\mathbb{R}} \int_{0}^{\infty} V(f, g)((r, x)(\mu, \lambda)) d \nu(r, x)\right) d \gamma(\mu, \lambda),
\end{aligned}
$$

and by Theorem 3.6

$$
\left[W_{\sigma_{1}}(g)\right](f)=c \iint_{\Gamma} \mathscr{F}_{\alpha}(f)(\mu, \lambda) d \gamma(\mu, \lambda) .
$$

We complete the proof by using relation (2.25). 
Remark 6.4. From Proposition 6.3, we deduce that there exists $\sigma \in \mathscr{Y}_{*}^{\prime}\left(\mathbb{R}^{2} \times \Gamma\right)$ given by a function in $L^{\infty}\left(\mathbb{R}^{2} \times \Gamma\right)$, such that for all $g \in \mathscr{Y}_{*}\left(\mathbb{R}^{2}\right)$ satisfying

$$
c=\int_{\mathbb{R}} \int_{0}^{\infty} g(r, x) d \nu(r, x) \neq 0,
$$

the distribution $W_{\sigma}(g)$ is not given by a function of $L^{2}(d v)$.

7. Weyl transform with symbol in $L^{p}(d \nu \otimes d \gamma), 2<p<\infty$

Theorem 7.1. Let $p \in] 2,+\infty\left[\right.$. There exists a function $\sigma \in L^{p}(d \nu \otimes d \gamma)$, such that the Weyl transform $W_{\sigma}$ defined by (6.1) is not a bounded linear operator on $L^{2}(d \nu)$.

We break down the proof into two lemmas, of which the theorem is an immediate consequence.

Lemma 7.2. Let $2<p<\infty$. Suppose that for all $\sigma \in L^{p}(d \nu \otimes d \gamma)$, the Weyl transform $W_{\sigma}$ given by relation (6.1) is a bounded linear operator on $L^{2}(d \nu)$. Then, there exists a positive constant $M$ such that

$$
\left\|W_{\sigma}\right\|_{*} \leqslant M\|\sigma\|_{p, \nu \otimes \gamma}, \quad \forall \sigma \in L^{p}(d \nu \otimes d \gamma) .
$$

Proof. Under the assumption of the lemma, there exists for each $\sigma \in L^{p}(d \nu \otimes d \gamma)$ a positive constant $C_{\sigma}$ such that

$$
\left\|W_{\sigma}(g)\right\|_{2, \nu} \leqslant C_{\sigma}\|g\|_{2, \nu}, \quad \text { for } g \in L^{2}(d \nu) .
$$

Let $f, g \in \mathscr{Y}_{*}\left(\mathbb{R}^{2}\right)$ such that $\|f\|_{2, v}=\|g\|_{2, v}=1$, and let us define the operator

$$
Q_{f, g}: L^{p}(d \nu \otimes d \gamma) \longrightarrow \mathbb{C}
$$

by

$$
Q_{f, g}(\sigma)=\left\langle\frac{W_{\sigma}(g)}{\bar{f}}\right\rangle
$$

Then,

$$
\sup _{\|f\|_{2, \nu}=\|g\|_{2, \nu}=1}\left|Q_{f, g}(\sigma)\right| \leqslant C_{\sigma} .
$$

By the Banach-Steinhauss theorem, the operator $Q_{f, g}$ is bounded on $L^{p}(d \nu \otimes d \gamma)$, then there exists a positive constant $M$ such that

$$
\left\|Q_{f, g}\right\|_{*}=\sup _{\|\sigma\|_{p, v \otimes \gamma}=1}\left|Q_{f, g}(\sigma)\right| \leqslant M .
$$

From this, we deduce that for all $f, g \in \mathscr{S}_{*}\left(\mathbb{R}^{2}\right)$, and $\sigma \in L^{p}(d \nu \otimes d \gamma)$, we have

$$
\left|\left\langle\frac{W_{\sigma}(g)}{\bar{f}}\right\rangle\right| \leqslant M\|\sigma\|_{p, \nu \otimes \gamma}\|f\|_{2, \nu}\|g\|_{2, \nu},
$$

which implies (7.1). 
Lemma 7.3. For $2<p<\infty$, there is no positive constant $M$ satisfying (7.1).

Proof. Suppose that there exists $M>0$ such that relation (7.1) holds.

Let $p^{\prime}$ be such that $1 / p+1 / p^{\prime}=1$, then $\left.p^{\prime} \in\right] 1,2[$.

We consider for $f, g \in \mathscr{I}_{*}\left(\mathbb{R}^{2}\right)$, the function $V(f, g)$ given by the relation (3.1). We have

$$
\|V(f, g)\|_{p^{\prime}, \nu \otimes \gamma}=\sup _{\|\sigma\|_{p, \nu \otimes \gamma}=1}\left|\left\langle\frac{W_{\sigma}(g)}{\bar{f}}\right\rangle\right| \leqslant \sup _{\|\sigma\|_{p, \nu \otimes \gamma}=1}\left\|W_{\sigma}(g)\right\|_{2, \nu}\|f\|_{2, \nu},
$$

and consequently

$$
\|V(f, g)\|_{p^{\prime}, \nu \otimes \gamma} \leqslant M\|f\|_{2, \nu}\|g\|_{2, \nu} .
$$

Now, let $f, g \in L^{2}(d \nu)$, we choose sequences $\left(f_{k}\right)_{k \in \mathbb{N}}$ and $\left(g_{k}\right)_{k \in \mathbb{N}}$ in $\mathscr{Y}_{*}\left(\mathbb{R}^{2}\right)$, approximating $f$ and $g$ in the $\|\cdot\|_{2, \nu}$-norm.

From (7.9), we get

$$
\left\|V\left(f_{k}, g_{k}\right)\right\|_{p^{\prime}, v \otimes \gamma} \leqslant M\left\|f_{k}\right\|_{2, \nu}\left\|g_{k}\right\|_{2, \nu}
$$

which implies that $\left(V\left(f_{k}, g_{k}\right)\right)_{k \in \mathbb{N}}$ is a Cauchy sequence in $L^{p^{\prime}}(d \nu \otimes d \gamma)$. Then, it converges to some function $F$ in $L^{p^{\prime}}(d \nu \otimes d \gamma)$.

Now, using Proposition 3.3, we deduce that $F=V(f, g)$, and

$$
\forall f, g \in L^{2}(d \nu), \quad\|V(f, g)\|_{p^{\prime}, \nu \otimes \gamma} \leqslant M\|f\|_{2, \nu}\|g\|_{2, \nu} .
$$

We will exhibit an example where the relation (7.11) leads to a contradiction. Let $f$ be defined on $\mathbb{R}^{2}$, even with respect to the first variable, and supported in $[-1,1] \times[-1,1]$. Then, for all $((r, x),(\mu, \lambda)) \in \mathbb{R}^{2} \times \Gamma$,

$$
|V(f, f)((r, x),(\mu, \lambda))| \leqslant|f| *|\check{f}|(r,-x)
$$

where $*$ is the convolution product given by Definition 2.2. From (2.18), we deduce that for all $(\mu, \lambda) \in \Gamma$, the function $(r, x) \mapsto V(f, f)((r, x),(\mu, \lambda))$ is supported in $[-2,2] \times$ $[-2,2]$.

On the other hand, by Hölder's inequality, we have

$$
\begin{aligned}
\left(\iint_{\Gamma} \mid\right. & \left.\left.\int_{-2}^{2} \int_{0}^{2} V(f, f)((r, x),(\mu, \lambda)) d \nu(r, x)\right|^{p^{\prime}} d \gamma(\mu, \lambda)\right)^{1 / p^{\prime}} \\
& \leqslant\left(\int_{-2}^{2} \int_{0}^{2} d \nu(r, x)\right)^{1 / p}\left(\iint_{\Gamma} \int_{-2}^{2} \int_{0}^{+\infty}|V(f, f)((r, x),(\mu, \lambda))|^{p^{\prime}} d \nu(r, x) d \gamma(\mu, \lambda)\right)^{1 / p^{\prime}} \\
& =\left(\int_{-2}^{2} \int_{0}^{2} d \nu(r, x)\right)^{1 / p}\|V(f, f)\|_{p^{\prime}, \nu \otimes \gamma} \leqslant M\left(\int_{-2}^{2} \int_{0}^{2} d \nu(r, x)\right)^{1 / p}\|f\|_{2, \nu}^{2} .
\end{aligned}
$$


The last inequality follows from (7.9). Now, Theorem 3.6 implies that the function

$$
(\mu, \lambda) \longmapsto \int_{\mathbb{R}} \int_{0}^{+\infty} V(f, f)((r, x),(\mu, \lambda)) d \nu(r, x)=c \mathscr{F}_{\alpha}(f)(\mu, \lambda)
$$

belongs to $L^{p^{\prime}}(d \gamma)$, here $c=\int_{\mathbb{R}} \int_{0}^{+\infty} f(r, x) d \nu(r, x)$.

If we pick $c=\int_{\mathbb{R}} \int_{0}^{+\infty} f(r, x) d \nu(r, x) \neq 0$, and the last inequality, we deduce that the function $\mathscr{F}_{\alpha}(f)$ belongs to $L^{p^{\prime}}(d \gamma)$, and

$$
\left\|\mathscr{F}_{\alpha}(f)\right\|_{p^{\prime}, \gamma} \leqslant \frac{M}{|c|}\left(\int_{-2}^{2} \int_{0}^{2} d \nu(r, x)\right)^{1 / p}\|f\|_{2, \nu}^{2}
$$

In the following, we consider the particular function $f$ given by

$$
f(r, x)=|r|^{\beta} \mathbf{1}_{[-1,1]}(r) \mathbf{1}_{[-1,1]}(x),
$$

where $\mathbf{1}_{[-1,1]}$ is the characteristic function of the interval $[-1,1]$.

This function belongs to $L^{1}(d \nu) \cap L^{2}(d \nu)$, for $\beta>-(\alpha+1)$, and we have

$$
\widetilde{\mathscr{F}}_{\alpha}(f)(\mu, \lambda)=\frac{1}{2^{\alpha-1} \Gamma(\alpha+1) \sqrt{2 \pi}} \frac{\sin \lambda}{\lambda} \int_{0}^{1} r^{\beta+2 \alpha+1} j_{\alpha}(r \mu) d r,
$$

so

$$
\left\|\tilde{\mathscr{F}}_{\alpha}(f)\right\|_{p^{\prime}, \nu}^{p^{\prime}}=\frac{2^{p^{\prime}}}{\left(2^{\alpha} \Gamma(\alpha+1) \sqrt{2 \pi}\right)^{p^{\prime}+1}} \int_{\mathbb{R}}\left|\frac{\sin \lambda}{\lambda}\right|^{p^{\prime}} d \lambda \times \int_{0}^{+\infty}\left|\int_{0}^{1} r^{\beta+2 \alpha+1} j_{\alpha}(r \mu) d r\right|^{p^{\prime}} \mu^{2 \alpha+1} d \mu .
$$

However

$$
\int_{0}^{1} r^{\beta+2 \alpha+1} j_{\alpha}(r \mu) d r=\frac{1}{\mu^{\beta+2 \alpha+2}} \int_{0}^{\mu} r^{\beta+2 \alpha+1} j_{\alpha}(r) d r .
$$

Using the asymptotic expansion of $j_{\alpha}$ (see $\left.[7,12]\right)$, given by

$$
j_{\alpha}(r)=\frac{2^{\alpha+1 / 2} \Gamma(\alpha+1)}{\sqrt{\pi} r^{\alpha+1 / 2}}\left[\cos \left(r-\alpha \frac{\pi}{2}-\frac{\pi}{4}\right)+O\left(\frac{1}{r}\right)\right], \quad \text { as }(r \longrightarrow+\infty),
$$

we deduce that for $-(\alpha+1)<\beta<-(\alpha+1 / 2)$, the integral

$$
a=\int_{0}^{+\infty} r^{\beta+2 \alpha+1} j_{\alpha}(r) d r
$$


exists and is finite. This involves that

$$
\int_{0}^{1} r^{\beta+2 \alpha+1} j_{\alpha}(r \mu) d r \sim \frac{a}{\mu^{\beta+2 \alpha+2}}, \quad \text { as }(\mu \longrightarrow+\infty) .
$$

Then, there exist $A, B>0$ such that for

$$
\mu>A, \quad\left|\int_{0}^{1} r^{\beta+2 \alpha+1} j_{\alpha}(r \mu) d r\right| \geqslant \frac{B}{\mu^{\beta+2 \alpha+2}} .
$$

Replacing in relation (7.18), we get

$$
\left\|\widetilde{\mathscr{F}}_{\alpha}(f)\right\|_{p^{\prime}, \gamma}^{p^{\prime}} \geqslant \frac{(2 B)^{p^{\prime}}}{\left(2^{\alpha} \Gamma(\alpha+1) \sqrt{2 \pi}\right)^{p^{\prime}+1}} \int_{\mathbb{R}}\left|\frac{\sin \lambda}{\lambda}\right|^{p^{\prime}} d \lambda \int_{A}^{+\infty} \frac{d \mu}{\mu^{p^{\prime}(2 \alpha+\beta+2)-2 \alpha-1}} .
$$

Thus, for $\beta<-(2 \alpha+2)+\left(2 \alpha+2 / p^{\prime}\right)$,

$$
\left\|\mathscr{F}_{\alpha}(f)\right\|_{p^{\prime}, \gamma}^{p^{\prime}}=\left\|\widetilde{\mathscr{F}}_{\alpha}(f)\right\|_{p^{\prime}, \nu}^{p^{\prime}}=+\infty .
$$

This shows that relation (7.15) is false if we pick

$$
\beta \in]-(\alpha+1), \min \left(-\left(\alpha+\frac{1}{2}\right),-(2 \alpha+2)+\frac{2 \alpha+2}{p^{\prime}}\right)[.
$$

\section{References}

[1] C. Baccar, N. B. Hamadi, and L. T. Rachdi, Inversion formulas for Riemann-Liouville transform and its dual associated with singular partial differential operators, International Journal of Mathematics and Mathematical Sciences 2006 (2006), Article ID 86238, 26 pages.

[2] L. Debnath and D. Bhatta, Integral Transforms and Their Applications, 2nd ed., CRC Press, Florida, 2006.

[3] J. A. Fawcett, Inversion of N-dimensional spherical averages, SIAM Journal on Applied Mathematics 45 (1985), no. 2, 336-341.

[4] G. B. Folland, Real Analysis. Modern Techniques and Their Applications, Pure and Applied Mathematics (New York), John Wiley \& Sons, New York, 1984.

[5] H. Hellsten and L.-E. Andersson, An inverse method for the processing of synthetic aperture radar data, Inverse Problems 3 (1987), no. 1, 111-124.

[6] M. Herberthson, A numerical implementation of an inverse formula for CARABAS raw data, Internal Report D 30430-3.2, National Defense Research Institute, FOA, Linköping, 1986.

[7] N. N. Lebedev, Special Functions and Their Applications, Dover, New York, 1972.

[8] R. Ma and L. Peng, Weyl-Jacobi Transform, preprint, 2005.

[9] M. M. Nessibi, L. T. Rachdi, and K. Trimeche, Ranges and inversion formulas for spherical mean operator and its dual, Journal of Mathematical Analysis and Applications 196 (1995), no. 3, 861884.

[10] E. M. Stein, Interpolation of linear operators, Transactions of the American Mathematical Society 83 (1956), no. 2, 482-492.

[11] E. M. Stein and G. Weiss, Introduction to Fourier Analysis on Euclidean Spaces, Princeton University Press, New Jersey, 1971. 
[12] G. N. Watson, A Treatise on the Theory of Bessel Functions, 2nd ed., Cambridge University Press, London, 1966.

[13] H. Weyl, The Theory of Groups and Quantum Mechanics, Dover, New York, 1950.

[14] M. W. Wong, Weyl Transforms, Universitext, Springer, New York, 1998.

[15] J. Zhao and L. Peng, Wavelet and Weyl transforms associated with the spherical mean operator, Integral Equations and Operator Theory 50 (2004), no. 2, 279-290.

N. B. Hamadi: Department of Mathematics, Faculty of Sciences of Tunis, University Tunis, El Manar 2092, Tunis, Tunisia

E-mail address: nadia.zouari@edunet.tn

L. T. Rachdi: Department of Mathematics, Faculty of Sciences of Tunis, University Tunis, El Manar 2092, Tunis, Tunisia

E-mail address: lakhdartannech.rachdi@fst.rnu.tn 


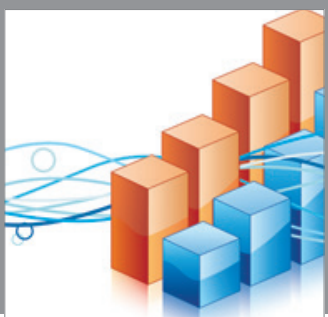

Advances in

Operations Research

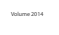

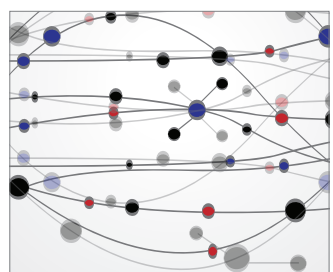

\section{The Scientific} World Journal
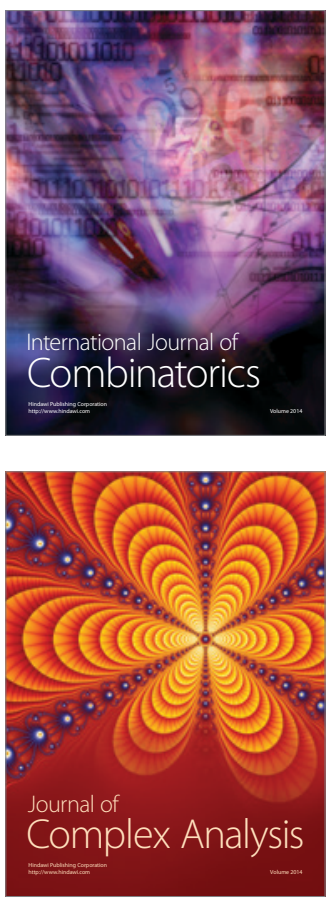

International Journal of

Mathematics and

Mathematical

Sciences
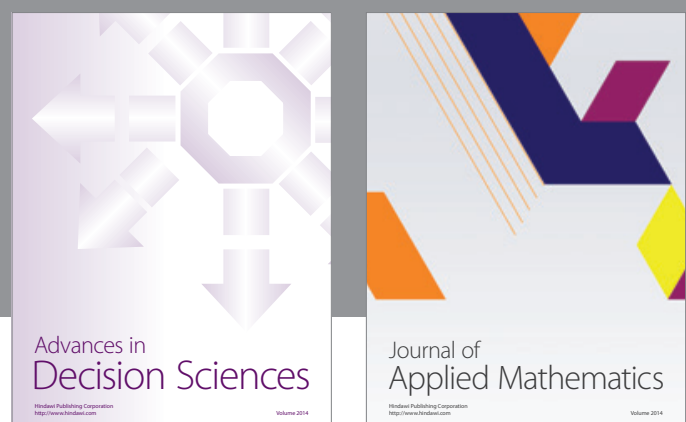

Journal of

Applied Mathematics
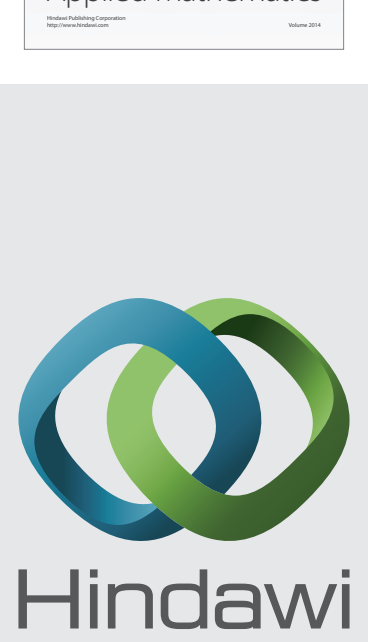

Submit your manuscripts at http://www.hindawi.com
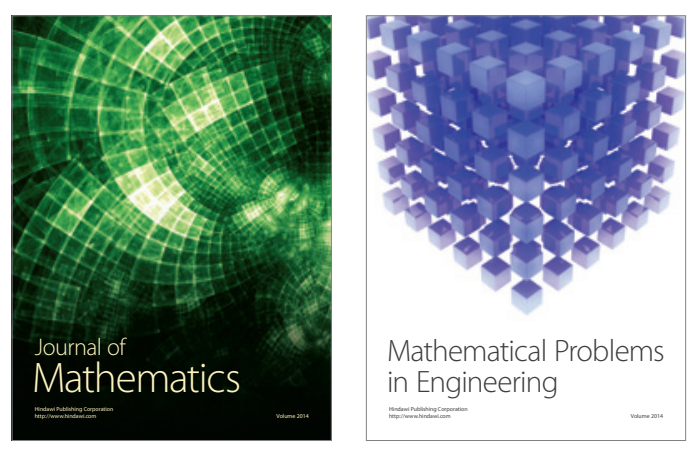

Mathematical Problems in Engineering
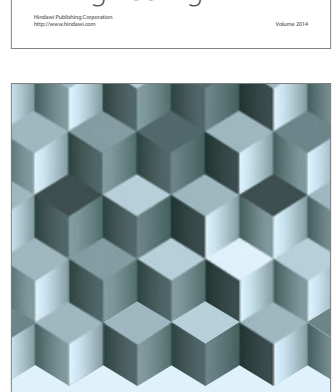

Journal of

Function Spaces
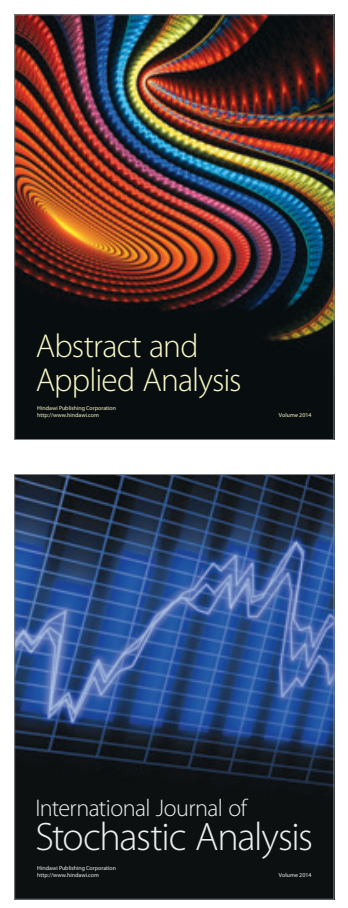

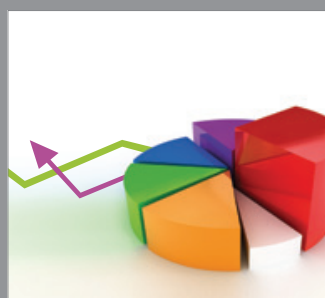

ournal of

Probability and Statistics

Promensencen
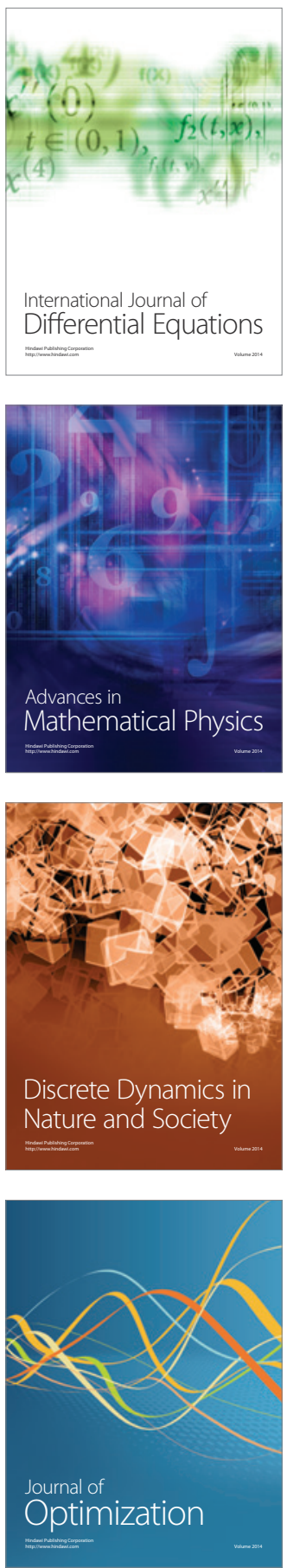